\title{
A relação entre sífilis congênita e o tratamento do parceiro da gestante: um estudo epidemiológico
}

A relationship between congenital syphilis and treatment of the pregnant woman's partner: an epidemiological study

\section{A Una relación entre la sífilis congénita y el tratamiento de la pareja de la mujer embarazada: un estudio epidemiológico}

\section{Renata de Souza Monteiro ${ }^{1}$, Paula Pitta de Resende Côrtes ${ }^{2}$}

Como citar esse artigo. Monteiro, RS; Côrtes, PPR. A relação entre sífilis congênita e o tratamento do parceiro da gestante: um estudo epidemiológico. Revista Pró-UniverSUS. 2019 Jul/Dez.; 10 (2): 13-17

\section{Resumo}

A sífilis congênita é uma doença infectocontagiosa responsável por desfechos desfavoráveis e custos para saúde pública. O rastreamento pré-natal sorológico é a medida mais importante para identificar os fatores de risco para sífilis congênita e a infecção transplacentária pode ocorrer em qualquer fase da gravidez. O presente artigo tem como objetivo demonstrar a relação do aumento de casos de sífilis congênita com o não tratamento dos parceiros de gestantes portadoras de sífilis. Esse estudo é transversal descritivo demonstrando um aumento do número de casos de sífilis congênita quando não há tratamento do parceiro da gestante, ainda que ela tenha realizado o tratamento, no Estado do Rio de Janeiro, no período de janeiro de 2013 a dezembro de 2017. A plataforma DATASUS foi utilizada para obtenção dos dados. Através dos dados, nota-se que em gestantes com prénatal realizado e parceiros tratados, o ano de menor incidência de sífilis congênita foi 2013 com um total de 412 casos e o ano com o maior número de casos foi 2015 com um total de 831. Em 2017, a queda foi mais significativa, reduzindo pela metade o total de casos se comparado a 2016, com um valor total de 447. Já em comparação às gestantes cujos parceiros não foram tratados, no período compreendido entre 2013 a 2016 a incidência de sífilis congênita foi ascendente. Este estudo mostrou a importância de um pré-natal bem realizado para detecção de gestantes portadoras de sífilis, assim como seu seguimento e o tratamento do seu parceiro.

Palavras-chave: Sífilis congênita, Pré natal, Gravidez, Prevenção.

\begin{abstract}
Congenital syphilis is an infectious disease responsible for unfavorable and public health costs. Serological prenatal screening is the most important measure for identifying risk factors for congenital syphilis and transplacental infection can occur at any other stage of pregnancy. This article aims to demonstrate the relationship between the increase in congenital syphilis cases and the non-treatment of partners of pregnant women with syphilis. This is a descriptive cross-sectional study showing an increase in the number of cases of congenital syphilis when there is no treatment of the pregnant woman's partner, even though she had the treatment, in the state of Rio de Janeiro, from January 2013 to December 2017. The DATASUS platform was used to obtain data. The data show that in pregnant women with prenatal care performed e and partners treated, the year with the lowest incidence of congenital syphilis was 2013 with a total of 412 cases and the year with the highest number of cases was 2015 with a total of 831 cases. In 2017, the drop was more significant, halving the total number of cases compared to 2016, with a total value of 447. Compared to pregnant women whose partners were not treated, in the period from 2013 to 2016 the incidence of congenital syphilis was rising. This study showed the importance of a well-performed prenatal care for the detection of pregnant women with syphilis, as well as its follow-up and the treatment of its partner.
\end{abstract}

Keywords: Congenital syphilis, Prenatal care, Pregnancy, Prevention.

2. Doutora. Professora do Curso de Medicina, Universidade de Vassouras. RJ, Brasil. Email: paulapitta@yahoo.com.br ORCID: https://orcid.org/0000-0001-5935-7045 


\section{Resumen}

La sífilis congénita es una enfermedad infecciosa responsable de resultados desfavorables y costos de salud pública La detección prenatal serológica es la medida más importante para identificar los factores de riesgo de sífilis congénita y la infección transplacentaria puede ocurrir en cualquier etapa del embarazo. Este artículo tiene como objetivo demostrar la relación entre el aumento de los casos de sífilis congénita y el no tratamiento de las parejas de mujeres embarazadas con sífilis. Este es un estudio descriptivo de corte transversal que muestra un aumento en el número de casos de sífilis congénita cuando no hay tratamiento de la pareja de la mujer embarazada, a pesar de que recibió el tratamiento en el estado de Río de Janeiro, desde enero de 2013 hasta diciembre de 2017. La plataforma DATASUS se utilizó para obtener datos. Los datos muestran que en mujeres embarazadas con atención prenatal y parejas tratadas, el año con la menor incidencia de sífilis congénita fue 2013 con un total de 412 casos y el año con el mayor número de casos fue 2015 con un total de 412 casos. 831. En 2017, la caída fue más significativa, reduciendo a la mitad el número total de casos en comparación con 2016, con un valor total de 447. En comparación con las mujeres embarazadas cuyas parejas no fueron tratadas, en el período comprendido entre 2013 y 2016 , la incidencia de sífilis congénita fue ascendente. Este estudio mostró la importancia de una atención prenatal bien realizada para detectar mujeres embarazadas con sífilis, así como su seguimiento y tratamiento de su pareja

Palabras clave: Sífilis congénita, Cuidado prenatal, Embarazo, Prevención.

\section{Introdução}

A Sífilis Congênita (SC) é uma doença causada pela bactéria espiroqueta Treponema pallidum de notificação compulsória, considerada indicador da qualidade da atenção materno-infantil. A SC se associa a um baixo grau de escolaridade, piores condições socioeconômicas, história anterior de risco obstétrico, início tardio do acompanhamento pré-natal e número insuficiente de consultas, assim como ao manejo inadequado dos casos com perda de oportunidade tanto para o diagnóstico quanto para o tratamento, à ausência de aconselhamento, à falta tratamento do parceiro e ao tratamento inadequado dos casos diagnosticados. ${ }^{1}$ Ainda que a prevalência da infecção pela sífilis tenha diminuído progressivamente com a descoberta da penicilina nos anos 40 tem-se observado tendência mundial no retorno da sífilis (SF) entre a população em geral e, de forma particular, dos casos de SC, tornando-a um dos mais desafiadores problemas de saúde pública. ${ }^{2}$

O contato sexual é a principal via de transmissão da sífilis, seguida pela transmissão vertical, esta última, passada para o feto durante a gestação de uma mãe com SF não tratada ou inadequadamente tratada. ${ }^{3}$

A via transplacentária é a forma mais comum de transmissão vertical e pode ocorrer em qualquer fase gestacional, sobretudo no terceiro trimestre, pois o fluxo placentário está mais desenvolvido. Contudo, quando a infecção se dá nos últimos meses tende a haver menor morbimortalidade, em função da maior capacidade de produzir resposta imunitária do feto. Eventualmente, identifica se outras formas menos comuns de aquisição da sífilis no início da vida, consideradas sífilis adquirida devido à deglutição de líquido amniótico contaminado e contato com lesão materna genital, no canal do parto, ou mamária, o que justificaria manifestações tardias da sífilis meses após o parto de crianças que eram soronegativas ao nascimento. ${ }^{1,3}$

As taxas de transmissão perinatal de sífilis estão, também, relacionadas à treponemia materna, quanto mais recente a infecção materna, maior o número de bactérias circulantes na corrente sanguínea aumentando o risco de transmissão e a gravidade do quadro. ${ }^{1,3}$

Quando a sífilis se manifesta logo após o nascimento ou pelo menos antes dos dois primeiros anos de vida, é denominada sífilis congênita precoce, sendo equivalente a sífilis secundária adquirida. Já a SC tardia diz respeito ao quadro iniciado após os dois anos e equivale à sífilis terciária adquirida. ${ }^{1}$

Nos quadros mais graves de sífilis congênita precoce tende a aparece icterícia, anemia e lesões cutâneos mucosas, sendo as mais comuns: exantema maculoso na face e extremidades, lesões bolhosas, condiloma latum, fissuras periorais e anais. A mucosa nasal pode apresentar rinite mucossanguinolenta com produção de muco rico em treponema. Além disso, em outros órgãos observa-se hepatoesplenomegalia (em função da colestase, fibrose e eritropoese extramedular), linfadenomegalia generalizada, osteocondrite, periostite ou osteíte, comprometimento do sistema nervoso central e hidropsia fetal. ${ }^{1,4}$ A prematuridade e o baixo peso ao nascer também são sinais que podem se manifestar na sífilis congênita e estão relacionados diretamente com os óbitos fetais e perinatais. ${ }^{5}$

$\mathrm{Na}$ sífilis congênita tardia as lesões são geralmente irreversíveis e mesmo o tratamento intensivo é insatisfatório. Nesta fase pode se perceber a tríade de Hutchinson, que cursa com alterações na dentição (dentes de Hutchinson), ceratite intersticial e surdez labiríntica, por comprometimento do VIII par craniano. Há, também, presença de molares em amora, tíbia em sabre, nariz em sela, fronte olímpica, maxila curta e mandíbula proeminente, perfuração do palato duro (devido à rinite sifilítica), retardo mental, dentre outros. ${ }^{1,4}$

A sífilis congênita é uma doença que pode ser evitada através de práticas realizadas durante a assistência ao pré natal que são efetivas para a prevenção dos casos. O diagnóstico precoce da infecção materna ainda é a melhor forma de prevenção, sendo o teste sorológico de fundamental importância.

A recomendação da Organização Mundial da Saúde acatada pelo Ministério da Saúde do Brasil de reduzir as taxas de SC é feita através da solicitação 
de testes não treponêmicos (VDRL) no primeiro e terceiro trimestre de gestação. Porém, são encontradas muitas dificuldades visto que nem todos os municípios encontram se no mesmo grau de desenvolvimento em relação à saúde pública. Percebe se, então, que em muitas regiões do país ainda há dificuldade de acesso ao pré-natal e aos exames laboratoriais. O Ministério da saúde ressalta também a importância do tratamento do parceiro da gestante portadora da sífilis. ${ }^{2}$

A possibilidade de erradicação da SC encontrase no diagnóstico precoce e tratamento adequado da sífilis gestacional que, embora de fácil diagnóstico, tratamento barato e eficaz, ainda se mantêm como um grave problema de saúde pública. $\mathrm{O}$ fato de ainda ser uma doença prevalente em nosso meio social mostra falhas na assistência pré-natal, como problemas na realização do exame de triagem (VDRL), no tratamento adequado da gestante e principalmente no tratamento do parceiro. ${ }^{6,7}$

No Brasil, estudos mostram que a falta de tratamento dos parceiros sexuais é um dos principais entraves para o controle da sífilis congênita.

O principal fator responsável pela elevada incidência da sífilis congênita em todo o mundo é a assistência pré-natal inapropriada. Outros estudos também associam à doença a pobreza, infecção pelo HIV, abuso de drogas e subutilização do sistema de saúde. Os fatores de risco individuais incluem gestantes adolescentes, raça não branca, baixa escolaridade, história de doenças sexualmente transmissíveis (DST), história de sífilis em gestações anteriores, múltiplos parceiros e baixa renda. ${ }^{8}$

Cabe ressaltar que a baixa escolaridade é considerada um marcador de maior risco para exposição às doenças sexualmente transmissíveis, devido a um limitado entendimento da importância das medidas de prevenção. ${ }^{9}$ Embora não seja uma doença restrita às camadas menos favorecidas, a pouca escolaridade e baixa renda podem ser marcadores importantes de pouco acesso aos serviços de saúde. Consequentemente, a assistência pré natal inadequada contribui para a persistência da transmissão vertical da sífilis nessa população. ${ }^{10}$

O presente artigo tem como objetivo estabelecer a relação do aumento do número de casos de sífilis congênita com o não tratamento dos parceiros de gestantes portadoras de sífilis mesmo que elas tenham realizado o pré-natal adequadamente.

\section{Materiais e Métodos}

Trata se de um estudo epidemiológico transversal descritivo, cujos dados foram obtidos através de consulta a base de dados do Departamento de Informática do Sistema Único de Saúde (DATASUS) no endereço eletrônico (http://tabnet.datasus.gov.br), que foi acessado em 04/08/2018.

A população do estudo foi constituída por todos os casos de sífilis congênita em gestantes que realizaram adequadamente o pré natal. $\mathrm{O}$ artigo visa correlacionar o maior número de casos de sífilis congênita quando não há tratamento do parceiro da gestante em detrimento do menor número de casos quando o parceiro é tratado. Os dados aqui citados são do Estado do Rio de Janeiro, no período de janeiro de 2013 a dezembro de 2017.

Por se tratar de um banco de dados de domínio público, não foi necessário submeter o projeto ao Comitê de Ética em Pesquisa.

\section{Resultados}

Através da análise dos dados, nota se que em gestantes com o pré natal realizado adequadamente juntamente com o tratamento do parceiro o número de menor incidência de sífilis congênita foi em 2013 com um total de 412 casos e o ano com o maior número de casos foi 2015 com um total de 831. Em 2014 houve um aumento do número de casos comparado ao ano anterior, contabilizando 765. Já em 2016, houve queda irrelevante de apenas dois casos em relação a 2015. Em 2017, a queda foi mais significativa, reduzindo pela metade o total de casos se comparado a 2016, com um valor total de 447 .

Já em comparação às gestantes cujos parceiros não foram tratados, no período compreendido entre 2013 a 2016 a incidência de sífilis congênita foi ascendente. As variáveis mais extremas de casos foram de 1086 no ano de 2013 e 1723 eventos no ano de 2016. Nos anos de 2014 e 2015 os casos registrados de sífilis congênita foram respectivamente 1280 e 1391. Em 2017 houve redução da incidência, sendo contabilizados 985 casos. Esses dados podem ser observados na Tabela 1 .

Correlacionando os números totais obtêm se 9752 de casos de sífilis congênita no Estado do Rio de Janeiro entre janeiro de 2013 e dezembro de 2017, dos quais 3287 eventos estão relacionados a gestantes que realizaram o pré-natal e o tratamento de seu parceiro e 6465 casos correspondem a gestantes que não realizaram o tratamento de seus parceiros, apesar da realização correta do pré-natal. Essas informações estão exemplificadas na Figura 1.

\section{Discussão}

O controle da sífilis está baseado na interrupção da cadeia de transmissão e na prevenção de novos casos, evitando assim a propagação da doença, são necessários a detecção e o tratamento individualizado precoce e adequado. A introdução do teste rápido em parceiros de 
Tabela 1. Pré natal realizado com o tratamento do parceiro e incidência de sífilis, 2013-2017.

\begin{tabular}{|c|c|c|}
\hline ANO & $\begin{array}{c}\text { PRÉ - NATAL E PARCEIRO } \\
\text { TRATADO }\end{array}$ & $\begin{array}{c}\text { PRE - NATAL E PARCEIRO NÃO } \\
\text { TRATADO }\end{array}$ \\
\hline 2013 & 412 & 1086 \\
\hline 2014 & 765 & 1280 \\
\hline 2015 & 831 & 1391 \\
\hline 2016 & 829 & 1723 \\
\hline 2017 & 447 & 985 \\
\hline
\end{tabular}

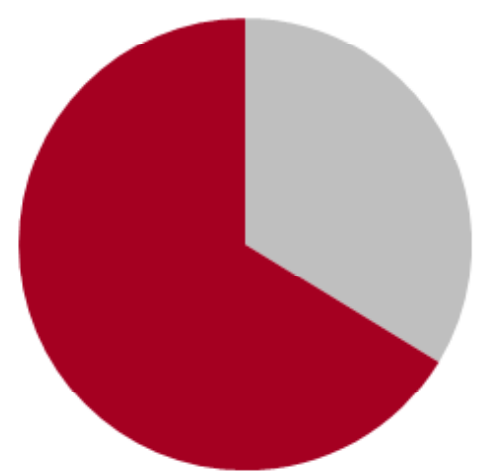

\author{
SC com parceiro \\ tratatado \\ - SC com parceiro não \\ tratatado
}

Figura 1. Gestantes que realizaram o pré-natal com parceiro tratado e não tratado, 20132017.

gestantes é muito importante, assim como o seu devido tratamento. ${ }^{11}$

As cinco décadas de experiência com a penicilina confirmam a sua absoluta superioridade no tratamento tanto da sífilis adquirida, em suas várias fases, como da congênita. A droga impede que as enzimas catalisadoras da formação de precursores da parede celular atuem. Com isso, não há restauração da parede, que é submetida continuamente à ação hidrolítica da lisozima produzida pelo organismo. A penicilina é, portanto, bactericida, desde que utilizada em doses e intervalos adequados. ${ }^{12}$

Quando se fala em tratamento da sífilis congênita, deve-se lembrar que a terapêutica da gestante com penicilina no primeiro trimestre costuma evitar a infecção fetal. ${ }^{12}$ A penicilina $\mathrm{G}$ benzatina é o fármaco de primeira escolha no tratamento da sífilis e o único indicado para gestantes: apresenta $98 \%$ de eficácia na prevenção da sífilis congênita, agindo em todos os estágios da doença. ${ }^{9}$

Também é importante levar em consideração que há alto risco de reinfecção se apenas as mulheres receberem o tratamento adequado e seus parceiros não; essas situações validam a importância do acompanhamento da gestante após o tratamento. Estudos também relatam a importância do tratamento do parceiro na cura da sífilis gestacional e prevenção da transmissão vertical. ${ }^{13}$

É fundamental reforçar a orientação sobre os riscos relacionados à infecção pelo T. pallidum por meio da transmissão sexual, para que as mulheres com sífilis e seus parceiros evitem relações sexuais ou mantenham práticas para o sexo seguro, como o uso de preservativo, durante o tratamento. Deve se recomendar o uso regular do preservativo (masculino ou feminino) também no período pós-tratamento. É indispensável que se inicie o tratamento imediatamente nos casos diagnosticados, tanto das gestantes quanto de seus parceiros e os tratamentos interrompidos devem ser reiniciados. ${ }^{14}$

Por fim, ressalta se que, em geral, a falha terapêutica está relacionada ao tratamento inadequado, 
ou seja, aquele realizado com outra medicação que não a penicilina; o uso de doses inadequadas de penicilina; o tratamento incompleto; em menor tempo que o recomendado; incorreto para a fase clínica da doença; ausência de documentação do tratamento anterior; ausência de queda dos títulos (controlado através do VDRL) após o tratamento adequado; parceiro não tratado, tratado inadequadamente ou quando não se tem tal informação. ${ }^{1,12}$

Dessa forma, reconhece se a importância da cura concomitante do parceiro como determinante para a cura da gestante.

\section{Conclusão}

A Sífilis congênita é uma doença de fácil prevenção e de diagnóstico e tratamento simples e se insere em um quadro de causa perinatal evitável, pois é possível realizar o tratamento efetivo na gestação.

Embora o diagnóstico e o tratamento sejam de fácil acesso e de baixo custo, a Sífilis congênita continua sendo um problema de saúde pública e deve continuar sendo alvo de estudos que gerem novas estratégias de prevenção. ${ }^{5}$ Por esse motivo, há necessidade de promover ações direcionadas ao controle dos casos, incluindo ações de notificação da doença, tratamento adequado dos parceiros sexuais e acompanhamento sorológico para comprovação da cura da doença.

Este estudo mostrou a importância de um pré-natal bem realizado para que se possa fazer a identificação das gestantes portadoras de sífilis e interromper o ciclo de transmissão. Contudo, apenas isso não é o suficiente, é de fundamental importância a busca ativa ao parceiro da gestante e o esclarecimento ao casal da necessidade do tratamento conjunto visto a redução considerável da incidência de sífilis congênita, quando o parceiro é tratado, se comparado as gestantes com parceiros não tratados. Dessa forma, evita se possíveis reinfecções impedindo a transmissão transplacentária.

Assim, é indiscutível a importância da educação em saúde não só para as gestantes, mas também para os seus parceiros sexuais para que seja possível diminuir a incidência do número de sífilis congênita.

\section{Referências}

1. Feitosa JAS, Rocha CHR, Costa FS. Artigo de Revisão. Rev. MedSaude Brasília. [Internet]. 2016 [acesso em jul 17]; 5(2): 286-97. Disponível em: https://portalrevistas.ucb.br/index.php/rmsbr/article/view/6749/4573

2. De Lorenzi DRS, Madi JM. Sífilis Congênita como Indicador de Assistência Pré-natal. Rev. Bras. Ginecol. Obstet. [Internet]. 2001 [acesso em 2019 Jul 17]; 23(10): 647-652. Disponível em: http://www.scielo.br/ scielo.php?script $=$ sci arttext\&pid=S0100-72032001001000006\&lng=en. http://dx.doi.org/10.1590/S0100-72032001001000006.

3. Guerra HS, Costa CV, Santos IAB, Silva JMS, Barcelos TB. Sífilis congênita: Repercussões e desafios. ArqCatarinMed [Internet]. 2017; [Acesso em 2019 Jul 17]; 46(3): 194-202. Disponível em: http://www.acm. org.br/acm/seer/index.php/arquivos/article/view/94
4. Avelleira JCR, Bottino G. Sífilis: diagnóstico, tratamento e controle. An. Bras. Dermatol. [Internet]. 2006 [acesso em 2019 Jul 17]; 81(2): 111-126. Disponível em: http:/www.scielo.br/scielo.php?script=sci arttext\&pid=S0365-05962006000200002\&lng=pt. http://dx.doi. org/10.1590/S0365-05962006000200002.

5. Sonda EC, Richter FF, Boschetti G, Casasola MP, Krumel CF, Machado $\mathrm{CPH}$. Sífilis congênita: uma revisão da literatura. Ver epidemiolcontrolinfect. [Internet]. 2013 [acesso em 2019 jul 17]; 3(1):28-30. Disponível em: https:// online.unisc.br/seer/index.php/epidemiologia/article/view/3022

6. Domingues RMSM, Saracen V, Hartz ZMA, Leal MC. Sífilis congênita: evento sentinela da qualidade da assistência pré-natal. Rev. Saúde Pública [Internet]. 2013 [acesso em 2019 Jul 17] ;47(1): 147-157. Disponível em: http://www.scielo.br/scielo.php?script=sci arttext\&pid=S0034 89102013000100019\&lng=en. http://dx.doi.org/10.1590/S003489102013000100019

7. Campos ALA, Araújo MAL, Melo SP, Andrade RFV, Gonçalves MLC. Sífilis em parturientes: aspectos relacionados ao parceiro sexual. Rev. Bras. Ginecol. Obstet. [Internet]. 2012 [acesso em 2019 Jul 17];34(9): 397-402. Disponível em: http://www.scielo.br/scielo.php?script=sci arttext\&pid=S0100-72032012000900002\&lng=pt. http://dx.doi. org/10.1590/S0100-72032012000900002.

8. Nonato SM, Melo APS, Guimarães MDC. Sífilis na gestação e fatores associados à sífilis congênita em Belo Horizonte- MG, 2010-2013. Epidemiol. Serv. Saúde [Internet]. 2015[acesso em 2019 Jul 17]; 24(4): 681-694. Disponível em: http://www.scielo.br/scielo.php?script=sci arttext\&pid=S2237-96222015000400681\&lng=pt. http://dx.doi. org/10.5123/S1679-49742015000400010.

9. Cavalcante PAM, Pereira RBL, Castro JGD. Sífilis gestacional e congênita em Palmas, Tocantins, 2007-2014. Epidemiol. Serv. Saúde [Internet]. 2017 [citado 2019 Jul 17]; 26(2): 255-264. Disponível em: http://www.scielo.br/ scielo.php?script $=$ sci_arttext\&pid=S2237-96222017000200255\&lng=pt. http://dx.doi.org/10.5123/s1679-49742017000200003.

10. Magalhães DMS, Kawaguchi IAL, Dias A, Calderon IMP. Sífilis materna e congênita: ainda um desafio. Cad. Saúde Pública [Internet]. 2013 [acesso em 2019 Jul 17] ;29(6): 1109-1120. Disponível em: http://www.scielo.br/ scielo.php?script $=$ sci_arttext\&pid=S0102-311X2013000600008\&lng=pt. http://dx.doi.org/10.1590/S0102-311X2013000600008.

11. Oliveira DR, Figueiredo MSN. Abordagem conceitual sobre a sífilis na gestação e o tratamento de parceiros sexuais. Enfermagem em foco [Internet]. 2011 [acesso em 2019 jul 17]; 2 (2):108-111. Disponível em: http://revista. cofen.gov.br/index.php/enfermagem/article/view/106

12. Guinsburg R, Santos AMN. Critérios diagnósticos e tratamento da sífilis congênita. São Paulo; Sociedade Brasileira de Pediatria. 2010

13. Padovani C, Oliveira RR, Pelloso SM. Sífilis na gestação: associação das características maternas e perinatais em região do sul do Brasil. Rev. Latino-Am. Enfermagem [Internet]. 2018 [acesso em 2019 Jul 17]; 26: e3019. Disponível em: http://www.scielo.br/scielo.php?script=sci arttext\&pid=S0104-11692018000100335\&lng=en. Epub Aug 09, 2018. http://dx.doi.org/10.1590/1518-8345.2305.3019.

14. SES-SP Secretaria de Estado da Saude. Serviço de Vigilância Epidemiológica; Coordenação do Programa Estadual DST/Aids-SP; Coordenadoria de Controle de Doenças CCD. Sífilis congênita e sífilis na gestação. Rev. Saúde Pública [online]. 2008, vol.42, n.4 [cited 2019-1119], pp.768-772. Disponível em: http://www.scielo.br/scielo.php?script=sci arttext\&pid=S0034-89102008000400026\&lng=en. 